\section{P827 A VERY HIGH PREVALENCE OF HUMAN PAPILLOMAVIRUS IN HIDDEN POPULATION OF SWINGERS: A DUTCH CASE-CONTROL STUDY}

${ }^{1}$ Fleur Koene ${ }^{*}$, ${ }^{2}$ Petra Wolffs, ${ }^{3}$ Nicole Dukers-Muijrers, ${ }^{4}$ Christian Hoebe. ${ }^{1}$ Amsterdam UMC and Maastricht UMC, Medical Microbiology, School of Public Health and Primary Care (CAPHRI), Amsterdam, Netherlands; '2Maastricht University Medical Center (MUMC), Medical Microbiology, Care and Public Health Research Institute (CAPHRI), Maastricht, Netherlands; ${ }^{3}$ Public Health Service South Limburg, Sexual Health, Infectious Diseases and Environmental Health, Heerlen, Netherlands; ${ }^{4}$ Public Health Service South Limburg, Maastricht University Medical Center (MUMC), Sexual Health, Infectious Diseases and Environmental Health, Medical Microbiology, Care and Public Health Research Institute (CAPHRI), Heerlen, Netherlands

\subsection{6/sextrans-2019-sti.872}

Background Swingers are at high risk for acquiring sexual transmitted infections (STI). The prevalence of human papillomavirus (HPV) in this population is unknown. This information is necessary to assess whether this high risk population should be included in the HPV prevention strategies. In this case-control study the prevalence of HPV in female swingers was tested.

Methods Vaginal swabs were collected from 94 female swingers (median age 45 years), visiting an STI clinic between October 2010 and December 2013 in South Limburg, the Netherlands. Ninety-three vaginal swabs from women visiting their general practitioner in this region, were matched in age and time of visit. Broad-spectrum HPV DNA amplification and mucosal HPV genotyping were performed using the highly sensitive SPF10 DEIA-LiPA25 system (DDL Diagnostic Laboratory, Rijswijk).

Results In 98\% of swingers HPV was detected compared to $18 \%$ of the matched controls. Of these HPV positive swingers, $75 \%$ carried at least one high risk type (hr-type). Twentyeight percent carried hr-types only, 25\% carried low risk types (lr-types) only and $47 \%$ carried one or more concurrent hrtypes and lr-types. There were no significant differences between the control group and the swingers in type of carriage (hr-type, lr-type, mixed). Hr-type 52 was most prevalent in swingers, followed by hr-types 31 and 53 (23\%). Hr-types 16 and 18 were detected in $15 \%$, respectively $11 \%$ of swingers. In the control group most prevalent were hr-types 16, 51 and 53 , detected in $18 \%$ of HPV positive women.

Conclusion In this study an unprecedented high HPV prevalence of $98 \%$ was found in female swingers, which was 5 times as much as in matched controls. Of the HPV positive swingers, a worrisome $75 \%$ carried at least one hr-type, detected with this highly sensitive detection method. These prevalence rates suggest a potential high HPV disease burden in this population and therefore targeted HPV prevention strategies are necessary.

Disclosure No significant relationships.

\section{P828 USE OF HORMONAL CONTRACEPTIVES AND GENITAL HUMAN PAPILLOMAVIRUS INFECTION: A NATIONAL MULTICENTER STUDY}

${ }^{1}$ Natalia Kops, ${ }^{1}$ Marina Bessel ${ }^{*},{ }^{2}$ Gerson Fernando Pereira, ${ }^{2}$ Flavia Souza, ${ }^{3}$ Adele Benzaken, ${ }^{4}$ Luisa Villa, ${ }^{4}$ Barbara Mello, ${ }^{1}$ Eliana Wendland. ${ }^{1}$ Hospital Moinhos de Vento, Porto Alegre, Brazil; ${ }^{2}$ Ministry of Health of Brazil, Department of Surveillance, Prevention and Control of STIS, HIVIAIDS and Viral Hepatitis, Brasilia, Brazil; ${ }^{3}$ Fundação de Medicina Tropical Doutor Heitor Vieira Dourado, Manaus, Brazil; ${ }^{4}$ Universidade de São Paulo and Instituto do Cancer do Estado de São Paulo, Faculdade De Medicina, São Paulo, Brazil

10.1136/sextrans-2019-sti.873
Background Available data suggest mixed evidence of hormonal contraceptives (HC) on the association with high-risk human papillomavirus (HPV) types, the main cause of cervical cancer. Therefore, we aimed to analyze the use of $\mathrm{HC}$ and the prevalence of HPV in unvaccinated Brazilians women aged 16-25 years.

Methods We analyzed cross-sectional data collected from 5,268 sexually active women who participated in the National Survey of Human Papillomavirus Prevalence (POP-Brazil). This survey enrolled participants from all 26 Brazilian capitals and the Federal District. Professionals from primary care units were trained for specimen and data collection. All participants answered a face-to-face questionnaire and provided biological samples for genital HPV analysis. HPV genotyping for HPV types was done using the Roche PCR-based Linear Array Genotyping Test. Sampling weights by sex and age were applied to the data.

Results The majority of the participants use any type of contraception $[76.58 \% \quad(95 \%$ CI 74.82 - 78.35)], being 2,577 [47.59\% (45.54\% - 49.63\%)] on hormonal contraceptives. The use of HC was more frequent in older women ( $\geq 22$ years), with higher education, married or living with a partner, and those with less than two sexual partners in the last year. No significant difference was found between the prevalence of high-risk HPV types and the use of HC: $38.57 \%$ among users of HC vs. $38.56 \%$ among don't users of HC ( $\mathrm{p}=0.994)$. Multivariate analysis showed that HC was not associated with high-risk HPV even after adjusting for age, educational level, presence of partner, smoke, and number of partners.

Conclusion Use of HC was not associated with HPV DNA detection. Even so, more studies are necessary evaluating the type of hormonal contraceptive, as well as length and dose of use.

Disclosure No significant relationships.

\section{P829 HUMAN PAPILLOMAVIRUS TYPES DISTRIBUTION IN HEAD AND NECK: A META-ANALYSIS FROM INFECTION TO CANCER}

${ }^{1}$ Yong Lu*, ${ }^{2}$ Huachun Zou. ${ }^{1}$ School of Public Health, Sun Yat-Sen University, Guangzhou, PR China, Guangzhou, China; ${ }^{2}$ School of Public Health (Shenzhen), Sun Yat-Sen University, Shenzhen, PR China, Guangzhou, China

\subsection{6/sextrans-2019-sti.874}

Background Human papillomavirus (HPV) has been shown to be involved in the carcinogenic mechanisms of non-genital malignancies such as head and neck cancers. Particular HPV types may preferentially progress from infection to cancer of head and neck. However, prospective data on the carcinogenesis process of head and neck cancer remain limited. We aimed to assess data on the relative carcinogenic potential of HPV that can inform head and neck cancer prevention through vaccination and screening.

Methods We systematically reviewed relevant literature in MEDLINE, Embase and Cochrane Library to identify studies published before April, 2018, which reported type-specific HPV prevalence at head and neck. We pooled type-specific HPV prevalence across the full spectrum of head and neck diagnoses, from normal cytology to head and neck cancer, stratifying anatomical site, gender, region and HIV status.

Results A total of 150 studies were included in our review, contributing 6985 cases of normal cytology, 1782 cases of head and neck lesions, and 13587 cases of head and neck 